$40 \mathrm{mg}$ twice daily, bismuth tripotassium dicitrate $300 \mathrm{mg}$ four times a day, tetracycline $500 \mathrm{mg}$ four times a day, and metronidazole $500 \mathrm{mg}$ three times a day, for 10 days. The primary end point was the eradication rate in the second-line treatment according to intention to treat (ITT) analysis. The minimum inhibitory concentrations were determined by agar dilution test.

Results The results were available for analysis in 398 patients up to Dec, 2017. The preliminary eradication rate in the EAML and BQ groups were 88.9\% (169/190) and 91\% (172/ $189)$, respectively $(\mathrm{p}=0.505)$ in the ITT analysis, and were $89.9 \%(169 / 188)$ and $96.1 \%(172 / 179)$ in the PP analyses, respectively $(\mathrm{p}=0.021)$ in the second line treatment. The efficacy of levofloxacin sequential therapy, but not bismuth quadruple therapy, appeared to be affected by levofloxacin resistance. In the third-line therapy, the eradication rate of EAML was $60 \%(3 / 5)$ for patients who failed after bismuth quadruple therapy. The eradication rate of BQ was $80 \%$ (12/ 15) for patients who failed after levofloxacin sequential therapy. The cumulative eradication rates were 95.3\% (181/190) and $92.6 \%(175 / 1189)$ in the EAML (2nd)-BQ(3rd) and the $\mathrm{BQ}(2 \mathrm{nd})-$ EAML (3rd) groups $(\mathrm{p}=0.276)$. The frequencies of adverse effects were $42.8 \%(62 / 145)$ and $81.9 \%(118 / 144)$ in patients treated with EAML and BQ, respectively $(\mathrm{p}<0.001)$. Conclusions Levofloxacin sequential therapy and bismuth quadruple therapy are similarly effective in the second-line treatment for $H$. pylori infection. (Trial registration number: NCT NCT03148366).

\section{IDDF2018-ABS-0220 FAECAL MICROBIOTA TRANSPLANTATION FOR MAINTENANCE OF CLINICAL REMISSION IN PATIENTS WITH ACTIVE ULCERATIVE COLITIS: A RANDOMISED CONTROL TRIAL}

Ramit Mahajan*, Vandana Midha, Varun Mehta, Arshdeep Singh, Husanpreet Khattar, Vikram Narang, Ajit Sood. Dayanand Medical College, India

\subsection{6/gutjnl-2018-IDDFbestabstracts. 15}

Background Though faecal microbiota transplantation (FMT) has been shown to be efficacious for induction of remission in patients with active UC, the long-term clinical outcomes after initial response have not been assessed.

Methods This single-blind, randomised, placebo-controlled trial was conducted at Dayanand Medical College and Hospital, India. Patients with active UC (Mayo score 4-10) who achieved clinical remission with multi-session $\operatorname{FMT}(0,2,6,10,14,18$ and 22 weeks) were randomly allocated in a $1: 1$ ratio to either maintenance FMT or placebo colonoscopic infusion every 8 weeks till 48 weeks. The primary endpoint was the maintenance of steroid-free clinical remission (Mayo score $\leq 2$ ), and secondary endpoints were the achievement of deep remission (clinical and endoscopic remission, i.e. endoscopic Mayo score 0) and histological remission (Nancy grade 0,1 ) at the end of 48 weeks. Clinical disease activity and adverse events were assessed at each visit or earlier in case of worsening of symptoms. The analysis was done by intention-to-treat and included all patients who underwent one session of FMT after initial clinical remission at week 22.

Results Forty-three of 78 patients treated with multi-session FMT achieved clinical remission, 22 of these were randomly assigned to receive FMT and 21 received placebo, colonoscopically every 8 weeks. The primary outcome was achieved in 19/22 (86.4\%) patients allocated FMT versus 14/21 $(66.7 \%)$ patients assigned placebo $(\mathrm{p}=0.126)$. Secondary endpoints of deep remission [18/22 (81.8\%) with FMT versus 8/ $21(38.1 \%)$ with placebo $\mathrm{p}=0.003]$ and histological remission [12/22 (54.5\%) with FMT versus 3/21 (14.3\%) with placebo $\mathrm{p}=0.006]$ were achieved in a significantly higher number of patients with FMT. Two patients receiving FMT and 5 patients on placebo relapsed. All relapses were treated with steroids. There were no serious adverse events necessitating discontinuation in patients on FMT, 1 patient who relapsed on placebo required colectomy.

Conclusions Maintenance therapy with FMT is required in patients who had earlier been treated with FMT for induction of remission. Moreover, FMT also enhances achievement of deep and histological remission.

\section{Clinical Hepatology}

\begin{tabular}{|l|l}
\hline IDDF2018-ABS-0058 & EARLY NORMALISATION OF ALANINE \\
& AMINOTRANSFERASE (ALT) AFTER \\
& NUCLEOS(T)IDE ANALOGUE TREATMENT \\
& REDUCES THE RISK OF HEPATOCELLULAR \\
& CARCINOMA (HCC) IN PATIENTS WITH \\
& CHRONIC HEPATITIS B - A TERRITORY- \\
& WIDE STUDY OF 21,182 SUBJECTS
\end{tabular}

${ }^{1}$ Terry Cheuk-Fung Yip*, ${ }^{2}$ Grace Lai-Hung Wong, ${ }^{1}$ Yee-Kit Tse, ${ }^{2}$ Henry Lik-Yuen Chan, ${ }^{2}$ Vincent Wai-Sun Wong. ${ }^{1} /$ Institute of Digestive Disease, Department of Medicine and Therapeutics, The Chinese University of Hong Kong, Hong Kong; ${ }^{2}$ Institute of Digestive Disease, Department of Medicine and Therapeutics, and State Key Laboratory of Digestive Disease, The Chinese University of Hong Kong, Hong Kong

\subsection{6/gutjnl-2018-IDDFbestabstracts. 16}

Background We aimed to evaluate the impact of alanine aminotransferase (ALT) normalisation (ALTN) achieved at different time after the start of antiviral treatment on the risk of hepatocellular carcinoma (HCC) in patients with chronic hepatitis B (CHB).

Methods We identified a territory-wide cohort of $\mathrm{CHB}$ patients who received entecavir and/or tenofovir disoproxil fumarate (TDF) for $\geq 1$ year between 2005 and 2016 in Hong Kong. Serial on-treatment ALT levels were analysed. ALTN referred to ALT level lower than the upper limit of normal (ULN) (30 U/L in males and $19 \mathrm{U} / \mathrm{L}$ in females). Early ALTN was defined as ALTN within 12 months. The primary outcome was HCC based on ICD-9-CM diagnosis codes. Patients with cancers previously or during the first year of treatment were excluded.

Results 21,182 CHB patients (10 437 with and 10745 without ALTN at 12 months after antiviral treatment) were identified and followed for a median (interquartile range) of 4.1 (2.4-6.0) years. Patients with or without ALTN at 12 months differed in gender distribution ( $76.9 \%$ vs. $58.4 \%$ male), baseline ALT (58 vs. $61 \mathrm{U} / \mathrm{L})$, baseline serum HBV DNA (4.9 vs. $\left.5.1 \log _{10} \mathrm{IU} / \mathrm{mL}\right)$, proportion of positive hepatitis $\mathrm{B}$ e antigen $(31.5 \%$ vs. $37.1 \%)$, and presence of cirrhosis $(8.8 \%$ vs. $10.5 \%)$ and diabetes mellitus (8.1\% vs. 9.1\%); 509 (2.4\%) patients developed HCC. ALTN at 3, 6, 9 and 12 months 